\title{
Coarse-Grained Back Reaction in Single Scalar Field Driven Inflation
}

\author{
Ghazal Geshnizjani \\ Department of Physics, Brown University, Providence, RI 02912, USA \\ Niayesh Afshordi \\ Princeton University Observatory, Princeton University, Princeton, NJ 08544, USA
}

\begin{abstract}
We introduce a self-consistent stochastic coarse-graining method, which includes both metric and scalar field fluctuations, to investigate the back reaction of long wavelength perturbations in single-scalar driven inflation, up to the second (one loop) order. We demonstrate that, although back reaction cannot be significant during the last 70 e-foldings of inflation with a smooth potential, there exist non-smooth inflaton potentials which allow significant back reaction, and are also consistent with cosmological observations. Such non-smooth potentials may lead to the generation of massive primordial black holes, which could be further used to constrain/verify these models.

PACS numbers: $98.80 . \mathrm{Cq}, 98.80 . \mathrm{Qc}$
\end{abstract}

\section{INTRODUCTION}

In the context of inflationary cosmology, initial seeds of today's structures are generated in the de-Sitter phase of an inflationary universe, as a consequence of quantum vacuum fluctuations (see e.g. 1] for a comprehensive overview of the theory of cosmological fluctuations and 2] for a recent introductory overview). The standard way of analyzing these fluctuations is through linear perturbation theory. However, the linear analyses is limited by the non-linear nature of the Einstein equations, and in particular, the presence of perturbations is likely to affect the evolution of the background cosmology at the nonlinear level. The back reaction of short wavelength gravity waves on an expanding Friedmann-Robertson-Walker (FRW) cosmology is a well-understood problem [3]. However, in the context of inflationary and post-inflationary cosmology, the scalar metric fluctuations (fluctuations coupled to energy density and pressure perturbations) are believed to dominate over the effects of gravity waves, and thus, it is of great interest to understand the possible back reaction of these scalar perturbations on the cosmological background. Furthermore, during inflation, the phase space of infrared modes (defined as modes with wavelength greater than the Hubble radius) grows exponentially, whereas the phase space of the ultraviolet modes does not grow and since the amplitude of the associated metric fluctuations of these infrared modes does not decrease in time, the back reaction of these infrared modes may grow to be significant. There have been different approaches to address this question. In [4, 5], the effective energy-momentum tensor formalism of 3 was generalized to study the back reaction of infrared modes on the spatially homogeneous component of the metric. The result was that, in a slow-roll inflationary background, the back reaction of infrared modes takes

*Electronic address: ghazal@het.brown.edu the form of a negative cosmological constant. This was later confirmed in [6] using very different techniques.

The objections (see 7]) to these analyses are mainly concerned with the fact that $3,[4,[5]$ do not consider a local observable quantity, and even if the effect is not simply a gauge artifact, it is still not clear how a local observer could distinguish the back reaction of longwavelength modes from an initial condition ambiguity in a homogenous universe. More importantly, calculating an "observable" from the spatially averaged metric will not in general give the same result as calculating the spatially averaged value of the observable. A later work by Abramo and Woodard in 8 tried to resolve this problem by identifying a local physical variable which describes expansion rate of the universe, then calculating the back reaction of cosmological perturbations on this quantity. A different approach was introduced in 9], where a simple variable describing the local expansion rate was considered and calculated up to second order in the metric fluctuations in a model with a single inflation field [28]. In this approach, the leading contribution of back reaction of infrared modes to this variable was evaluated. When evaluated at a fixed value of the matter field $\varphi$, the only physical clock available in this simple system, the dependence of the expansion rate on the clock time takes on exactly the same form as in an unperturbed background [29]. Thus, the leading infrared back reaction terms had no locally measurable effect in this system. Similar conclusions were reached in 12 .

However, the fact that during inflation the phase space of infrared modes is growing in time, i.e. modes are exiting the Hubble radius, leads us to the conjecture that there might be a locally measurable effect just due to these crossings, and if we include the corrections due this effect, the results for single scalar field models may change. Since these modes are generated in the quantum mechanical ultraviolet limit, handling them in general is very difficult. Attempts, so far, in order to model the evolution through writing a classical Langevin-like equation for the coarse-grained scaler field, the so-called 
stochastic approach 13], fail to include the inflaton and metric fluctuations self-consistently [30]. In this work, for the first time, we devise a self-consistent stochastic framework to study the evolution of the coarse-grained inflaton. While the approach uses the results of linear (metric+inflaton) perturbation theory for the ultraviolet and transitional modes, it is rigorously extended to the non-linear infrared regime, which allows a consistent study of the evolution of fluctuations through their entire history. We then apply this framework to the problem of back reaction in inflationary cosmology, and investigate how/when the non-linear effects may change properties/predictions of the standard slow-roll inflation.

The structure of the paper is as follows: In Section II, we explain how the linear theory of perturbations can be self-consistently generalized to the non-linear infrared regime, including both inflaton and metric fluctuations. We also introduce $\Theta$, the local expansion rate, and how it is related to metric perturbations. In Sec. III, we show that the quantum generation of fluctuations can be modelled as a stochastic term in the equation of motion for $\Theta$, coarse-grained over local Hubble patches. In Sec. IV, we apply this equation to a chaotic inflationary scenario with a quadratic potential $V(\varphi)=\frac{1}{2} m^{2} \varphi^{2}$ and evaluate the local expansion rate with back reaction corrections due to infrared modes. Sec. V contains the generalization of the result from Sec. IV to any power-law potential. In Sec. VI, we investigate if back reaction can ever significantly change the inflationary predictions for our observable universe. Finally, Sec. VII summarizes our results and concludes the paper. Throughout this paper, we use the natural units, and set the Planck mass equal to unity, i.e. $8 \pi G=c=\hbar=1$.

\section{GENERALIZED BARDEEN PARAMETER IN THE NONLINEAR REGIME}

Our analysis is based on the consistent quantization of the linearized metric and matter perturbations in a classical expanding background space-time. In this framework, it can be shown that, in the linear regime, the Sasaki-Mukhanov parameter $v$ [15, 16], defined as [31]:

$$
v=a\left[\delta \varphi+\left(\frac{\dot{\varphi}}{H}\right) \phi\right]=\frac{a \dot{\varphi}}{H} \zeta
$$

obeys the equation of motion of a free scalar field, on scales much smaller than the Hubble radius. Here, $\zeta$ is the Bardeen parameter [17]:

$$
\zeta=\phi-\frac{H}{\dot{H}}(H \phi+\dot{\phi}),
$$

where $\phi$ is the scalar metric perturbations in the longitudinal gauge, in a spatially flat universe:

$$
d s^{2}=(1+2 \phi) d t^{2}-a^{2}(t)(1-2 \phi) \delta_{i j} d x^{i} d x^{j} .
$$

We use the following ansatz for our non-linear metric:

$$
d s^{2}=e^{2 \phi} d t^{2}-e^{-2 \psi} \delta_{i j} d x^{i} d x^{j},
$$

which is a generalization of a metric with linear scalar perturbations. This ansatz, though not a general solution, represents the most important sector of the metric, as any such metric can be continuously connected to a metric with linear scalar perturbations, which is the dominant sector if perturbations are generated during slow-roll inflation [18]. We consider the simplest model of matter with a single scalar field $\varphi$, the inflaton. We also use a perfect fluid model to describe the expansion of the universe. The velocity four-vector field $u^{\alpha}$ can be considered to be tangential to a family of world lines of a matter fluid in a general space-time. This four-vector is normalized such that

$$
u^{\alpha} u_{\alpha}=1
$$

where $\alpha$ runs over the space-time indices. In terms of this four-vector, the local expansion rate $\Theta$ is defined by

$$
\Theta \equiv u_{; \alpha}^{\alpha}=3 H_{\text {local }},
$$

where $H_{\text {local }}$ is the local Hubble constant. It represents the local expansion rate of the tangential surfaces orthogonal to the fluid flow. It is easy to verify that, for our specific choice of gauge, $\Theta$ follows the following relation [32],

$$
\Theta=-3 \psi^{\prime},
$$

where a prime denotes the derivative with respect to $\tau$, the proper time, and is related to the coordinate time via $d \tau=e^{\phi} d t$. The Lagrangian and the energy-momentum tensor for the inflaton are given by:

$$
\begin{gathered}
\mathcal{L}=\frac{1}{2} \partial^{\alpha} \varphi \partial_{\alpha} \varphi-V(\varphi), \\
T_{\nu}^{\mu}=\partial^{\mu} \varphi \partial_{\nu} \varphi-\mathcal{L} \delta_{\nu}^{\mu} .
\end{gathered}
$$

It can be shown that for slow-roll inflation, in the longwavelength limit, $\Theta$ satisfies the following condition [9],

$$
\Theta=\sqrt{3 V(\varphi)} .
$$

In [19] it is demonstrated that, in these limits, the generalized Bardeen parameter:

$$
\tilde{\zeta}(\mathbf{x}) \equiv \psi-\frac{1}{2} \int\left(\frac{\partial \log (g)}{\partial \varphi}\right)^{-1} d \varphi
$$

remains constant in the classical non-linear regime [20]. Here, $g$ is a function of the inflaton field, obtained by integrating the $G_{0 i}$ Einstein constraint equations, and satisfies

$$
2 \psi^{\prime}=g(\varphi) ; \varphi^{\prime}=\frac{\partial g(\varphi)}{\partial \varphi}
$$


It is easy to see that for linear perturbations $\delta \tilde{\zeta}(x)$ reduces to the usual Bardeen parameter $\zeta$, which is known to remain constant for adiabatic super-Hubble modes 33.

Combining the above equation, with Eqs. (7) and ( 11), we see that

$$
\tilde{\zeta}^{\prime}=-\frac{\Theta}{3}-\frac{1}{2}\left(\frac{\partial \log (\Theta)}{\partial \varphi}\right)^{-1} \varphi^{\prime}=0,
$$

in the classical long-wavelength limit. In the next section, we show how quantum fluctuation affect Eq. (13).

\section{COARSE-GRAINING THE EXPANSION RATE}

In this section, we use coarse-graining on the scale of the Hubble radius to provide a framework to analyze back reaction, and obtain a new derivation of the equation of motion for stochastic inflation [21], which takes into account the metric fluctuations.

Let us define the coarse-grained generalized Bardeen parameter, $\zeta_{c}$, in the Fourier space, as:

$$
\tilde{\zeta}_{c}(\mathbf{k})=\theta(a H-k) \tilde{\zeta}(\mathbf{k})
$$

where $\theta$ is the step function (not to be mistaken with $\Theta$, the local expansion rate) which is zero for $k>a H$, and is one for $k \leq a H$ [34]. Taking the time derivative of the above equation; the time derivative of $\tilde{\zeta}$ vanishes for infrared modes and the time derivative of $\theta$ is a delta function at $k=a H$, which yields

$$
\tilde{\zeta}_{c}^{\prime}(\mathbf{k})=\tilde{\zeta}(\mathbf{k})\left(H+\frac{H^{\prime}}{H}\right) \delta\left(1-\frac{k}{a H}\right) .
$$

This simply implies that $\tilde{\zeta}_{c}^{\prime}(x)$ in real space is related to $\tilde{\zeta}(k)$ in the following way

$$
\tilde{\zeta}_{c}^{\prime}(\mathbf{x})=\left(H+\frac{H^{\prime}}{H}\right) \int \frac{\mathbf{d}^{3} \mathbf{k}}{(2 \pi)^{3}} e^{i \mathbf{k} \cdot \mathbf{x}} \tilde{\zeta}(\mathbf{k}) \delta\left(1-\frac{k}{a H}\right) .
$$

Two-point function of $\tilde{\zeta}(k)$ can be described in terms of its power spectrum, $P_{\tilde{\zeta}}(k)$, defined by:

$$
\left\langle\tilde{\zeta}\left(\mathbf{k}_{\mathbf{1}}\right) \tilde{\zeta}\left(\mathbf{k}_{\mathbf{2}}\right)\right\rangle=(2 \pi)^{3} \delta\left(\mathbf{k}_{\mathbf{1}}-\mathbf{k}_{\mathbf{2}}\right) P_{\tilde{\zeta}}\left(k_{1}\right) .
$$

Assuming that Hubble-scale perturbations are linear, $P_{\tilde{\zeta}}(k)$ can be derived in the linear theory of quantum generation of perturbations [1],

$$
P_{\tilde{\zeta}}(k) \simeq P_{\zeta}(k)=\left.\frac{H^{4}}{2 k^{3} \dot{\varphi}^{2}}\right|_{k=a H} .
$$

Using this knowledge for $\tilde{\zeta}(k)$ and assuming $H^{\prime} \ll H^{2}$ (the slow-roll condition) in the above equation, we can calculate the two-point function for $\tilde{\zeta}_{c}^{\prime}(\mathbf{x}, \tau)$. Note that
$H$ in Eqs. (14 18) is the local Hubble constant, and can be replaced by $\Theta / 3$. Combining Eqs. (16 18), after performing few simple integrations, we arrive at:

$$
\begin{aligned}
& \left\langle\tilde{\zeta}_{c}^{\prime}\left(\mathbf{x}_{\mathbf{1}}, \tau_{1}\right) \tilde{\zeta}_{c}^{\prime}\left(\mathbf{x}_{\mathbf{2}}, \tau_{2}\right)\right\rangle \\
= & -\frac{1}{648 \pi^{2}} \frac{\Theta^{5}}{\Theta^{\prime}} \delta\left(\tau_{1}-\tau_{2}\right) \operatorname{sinc}\left(a H\left|\mathbf{x}_{\mathbf{1}}-\mathbf{x}_{\mathbf{2}}\right|\right)
\end{aligned}
$$

where $\operatorname{sinc}(x)=\frac{\sin (x)}{x}$ and $\operatorname{sinc}(0)=1$. Let us express $\tilde{\zeta}_{c}^{\prime}$ as

$$
\tilde{\zeta}_{c}^{\prime}(x, \tau) \equiv \frac{1}{36 \pi}\left(\frac{-\Theta^{5}}{2 \Theta^{\prime}}\right)^{1 / 2} \xi(x, \tau)
$$

where the variable $\xi$ is a random Gaussian field with:

$$
<\xi\left(\mathbf{x}_{\mathbf{1}}, t_{1}\right) \xi\left(\mathbf{x}_{\mathbf{2}}, t_{2}\right)>=\delta\left(\tau_{1}-\tau_{2}\right) \operatorname{sinc}\left(a H\left|\mathbf{x}_{\mathbf{1}}-\mathbf{x}_{\mathbf{2}}\right|\right) .
$$

Now, plugging the coarse grained value of $\tilde{\zeta}^{\prime}$ from Eq. (20) into the left hand side of Eq. (13), we may obtain the coarse-grained field equation:

$$
\varphi^{\prime}=\frac{\partial \log (\Theta)}{\partial \varphi}\left[-\frac{2}{3} \Theta-\frac{\sqrt{2}}{36 \pi}\left(\frac{-\Theta^{5}}{\Theta^{\prime}}\right)^{\frac{1}{2}} \xi(x, t)\right] .
$$

\section{THE CASE OF A QUADRATIC POTENTIAL}

In this section, we apply the coarse-graining method, developed in the previous section, to a chaotic inflationary scenario with a quadratic potential, and investigate the evolution of the background during inflation under the effect of second-order back reaction.

Here, we assume that the potential for the inflaton field, $\varphi$, has a quadratic form:

$$
V(\varphi)=\frac{1}{2} m^{2} \varphi^{2}
$$

Plugging this into Eq. (10), and then (22), the equation of motion for $\Theta$ reduces to

$$
\Theta^{\prime}+m^{2}=\frac{\sqrt{2}}{24 \pi} m \Theta^{\frac{3}{2}} \xi(x, \tau) .
$$

This equation can be solved perturbatively, taking $\xi$ as the perturbation variable. $\Theta$ can be approximated by

$$
\Theta=\Theta_{0}+\Theta_{1}+\Theta_{2},
$$

where $\Theta_{0}, \Theta_{1}$ and $\Theta_{2}$ are zeroth, first and second order in $\xi$, respectively. As the first step in solving Eq. (24), we note that $\Theta_{0}$ is simply the expansion rate in the absence of perturbations,

$$
\Theta_{0}=-m^{2} \tau,
$$

where our convention for $\tau$ is so that it has a negative initial value $\tau_{i n}$ and goes to zero towards the end of inflation, so that $\Theta$ decreases as inflation proceeds. Now we substitute $\Theta_{0}$ back into Eq. (24) to find $\Theta_{1}$,

$$
\Theta_{1}=\frac{\sqrt{2}}{24 \pi} m \int\left(-m^{2} \tau\right)^{3 / 2} \xi(\mathbf{x}, \tau) d \tau
$$


This step is easily followed by calculation of $\Theta_{2}$.

$$
\Theta_{2}=\frac{m^{6}}{288 \pi^{2}} \int_{\tau_{i n}}^{\tau} \int_{\tau_{i n}}^{\tau_{1}} d \tau_{1} d \tau_{2}\left(\tau_{1} \tau_{2}^{3}\right)^{1 / 2} \xi\left(\mathbf{x}, \tau_{1}\right) \xi\left(\mathbf{x}, \tau_{2}\right)
$$

Since $\xi$ is a random Gaussian variable, we are not interested in the spontaneous value of $\Theta$, and instead investigate the statistical behavior of $\langle\Theta\rangle$, the expectation value of the expansion rate over time as more infrared modes cross the hubble radius and may build up a back reaction effect. As $\Theta_{0}$ does not have a $\xi$ term, and the expectation value of $\Theta_{1}$ vanishes over time, we have:

$$
\langle\Theta\rangle=\Theta_{0}+\left\langle\Theta_{2}\right\rangle
$$

where $\left\langle\Theta_{2}\right\rangle$ can be derived from Eqs. (28) and (21), which finally enables us to calculate expansion rate with corrections due to stochastic back reaction:

$$
\langle\Theta\rangle=-m^{2} \tau+\frac{m^{6}}{1728 \pi^{2}}\left(\tau^{3}-\tau_{\text {in }}^{3}\right) .
$$

Contrary to our expectation from [5], we can see that the back reaction term has a positive value and it is increasing with time, as if it is speeding up inflation. However, as we see in the next section, this is merely a result of our choice to average over hyper-surfaces of constant proper time, while other choices of time hyper-surfaces may lead to different forms, or even a different sign, for the back reaction term.

Nevertheless, as both $\Theta$ and $\tau$ are observable, we may conclude that the back reaction does not vanish during inflation and since its magnitude is increasing with time, it may become important if inflation lasts long enough. Notice that this correction is, in fact, due to wavelengths larger than the Hubble radius as, using Eq. (28), $\left\langle\Theta_{2}\right\rangle$ could be expressed as an integral over $k$, the wave number instead of $\tau$, which would yield

$$
\left\langle\Theta_{2}\right\rangle=\left.\frac{1}{594 \pi^{2}} \int d \ln k \cdot k^{3} P_{\zeta}(k) \cdot \frac{\Theta_{0}^{\prime 2}}{\Theta_{0}^{3}}\right|_{k},
$$

and the above integral is only over modes which have already crossed the Hubble radius. In this sense, this is an infrared back reaction effect.

\section{GENERALIZATION TO POWER LAW POTENTIALS}

In this section, we generalize the calculations of the last section to power law inflaton potentials. Also, rather than proper time, we use the inflationary e-folding number as our time variable, as it has more direct and intuitive observational relevance. The number of e-foldings since the beginning of inflation is

$$
N_{e}(\tau)=\frac{1}{3} \int_{\tau_{i n}}^{\tau} \Theta d \tau
$$

Using Eq. (7), we can see that $\psi$ may be used as a measure of e-folding:

$$
N_{e}(\tau)=\psi_{i n}-\psi(\tau)
$$

and thus, in order to find how $\Theta$ changes as a function of $N_{e}$, it is sufficient to express it in terms of $\psi$. Combining Eq. (19) with $\frac{d}{d \tau}=\psi^{\prime} \frac{d}{d \psi}$, we can re-write Eq. (22) as

$$
\frac{d \varphi}{d \psi}=\frac{\partial \log (\Theta)}{\partial \varphi}\left[2-\frac{\sqrt{2}}{6 \pi}\left(\frac{\Theta^{3}}{d \Theta / d \psi}\right)^{\frac{1}{2}} \xi(\mathbf{x}, \psi)\right]
$$

where $\xi$ in the above equation like is, again, a random Gaussian field, defined by

$<\xi\left(\mathbf{x}_{\mathbf{1}}, \psi_{1}\right) \xi\left(\mathbf{x}_{\mathbf{2}}, \psi_{2}\right)>=\delta\left(\psi_{1}-\psi_{2}\right) \operatorname{sinc}\left(a H\left|\mathbf{x}_{\mathbf{1}}-\mathbf{x}_{\mathbf{2}}\right|\right)$

In the case of single scalar field inflationary models with a power law potential

$$
V(\varphi)=M^{4} \varphi^{\alpha}
$$

using the procedure described in the previous section, Eq. (34) can be solved to obtain $\Theta$ in terms of $\psi$ :

$$
\begin{aligned}
\langle\Theta\rangle \quad & =\alpha^{\alpha / 4}\left(3 M^{4}\right)^{1 / 2} \psi^{\alpha / 4}+ \\
& \frac{\left(3 M^{4}\right)^{3 / 2} \alpha^{1+3 \alpha / 4}}{288 \pi^{2}(\alpha+4)} \psi^{\frac{\alpha}{4}-2}\left(\psi^{2+\alpha / 2}-\psi_{\text {in }}^{2+\alpha / 2}\right) .
\end{aligned}
$$

Note that we assumed $\psi \rightarrow 0$ towards the end of inflation. The result may be simplified for the case of a quadratic potential:

$$
\begin{aligned}
\langle\Theta\rangle & =\sqrt{2}\left(3 M^{4}\right)^{1 / 2} \psi^{1 / 2} \\
& +\frac{\left(3 M^{4}\right)^{3 / 2} \sqrt{2}}{432 \pi^{2}} \psi^{-3 / 2}\left(\psi^{3}-\psi_{i n}^{3}\right) .
\end{aligned}
$$

We see that, if averaged over the hyper-surfaces of constant e-folding rather than constant proper time, the second order back reaction has a negative sign, i.e. it slows down inflation. We again believe that this is a real effect, as both $\Theta$ and $N_{e}$ are observable parameters.

\section{DOES BACK REACTION CHANGE INFLATIONARY PREDICTIONS?}

Let us calculate the ratio of the second order term in Eq. (37) to the zeroth order term

$$
\frac{\left\langle\Theta_{2}\right\rangle}{\Theta_{0}} \simeq \frac{3 M^{4}}{288 \pi^{2}} \alpha^{1+\alpha / 2} \psi_{i n}^{2+\alpha / 2} \psi^{-2},
$$

if $\psi \ll \psi_{i n}$, close to the end of inflation. Currently, the most stringent constraints on the inflationary potential is due to the observations of the cosmic microwave background anisotropies by the WMAP satellite [22]. The 


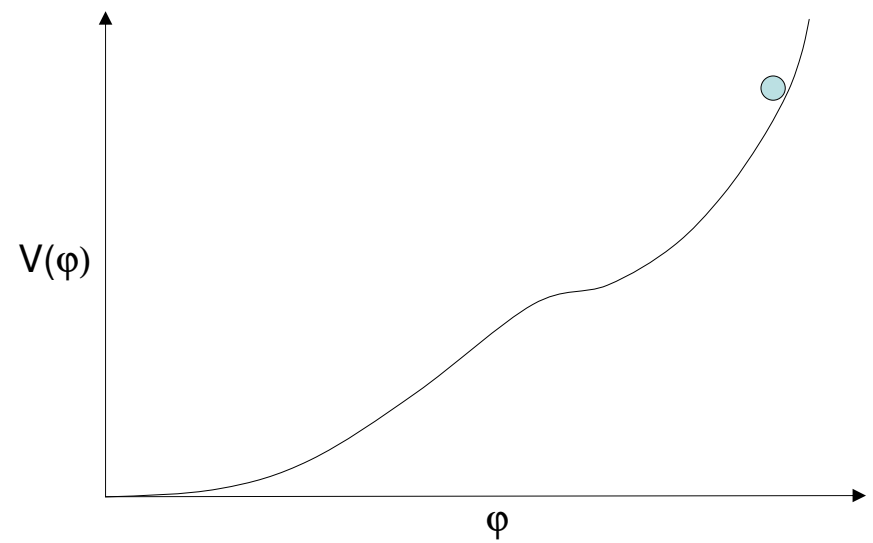

FIG. 1: The example of an inflaton potential with a flat portion. Back reaction can dominate the slow-roll inflation in this portion of the potential.

observed amplitude of anisotropies at the observable horizon scale require:

$$
\begin{aligned}
\Delta_{\mathcal{R}}^{2} \equiv \frac{k^{3} P_{\zeta}(k)}{2 \pi^{2}} & =\frac{V^{3}(\varphi)}{12 \pi^{2} V^{\prime 2}(\varphi)}=(2.1 \pm 0.3) \times 10^{-9}, \\
\text { for } k & =0.002 \mathrm{Mpc}^{-1}
\end{aligned}
$$

with $V^{\prime}$ being the derivative of $V$ with respect to $\varphi$. Then we can use the simple slow-roll prediction (first term in Eq. 38 along with Eq. 10) to relate $\varphi$ to $\psi$, and express $\Delta_{\mathcal{R}}^{2}$ in terms of $\psi$ :

$$
\Delta_{\mathcal{R}}^{2}=\frac{M^{4}}{12 \pi^{2}} \alpha^{\alpha / 2-1} \psi^{1+\alpha / 2} .
$$

Assuming that $\psi_{\text {in }} \simeq N_{e}$ (Eq. 33), and that inflation ends when $V^{\prime} \sim V$ (in Planck units), Eq. (39) can be expressed in terms of $\Delta_{\mathcal{R}}$ :

$$
\frac{\left\langle\Theta_{2}\right\rangle}{\Theta_{0}} \simeq \frac{1}{8} \Delta_{\mathcal{R}}^{2} N_{e}=2 \times 10^{-8}\left(\frac{\Delta_{\mathcal{R}}^{2}}{2 \times 10^{-9}}\right)\left(\frac{N_{e}}{70}\right) .
$$

Although, this result is specific to the power law inflaton potentials, we expect a similar order of magnitude for any smooth potential that is consistent with observational bounds. Does this mean that back reaction will always be negligible during inflation? Of course, the number of e-foldings, $N_{e}$, could be arbitrarily large. Indeed, after enough e-foldings, we end up with the selfsimilar chaotic inflationary picture which results from the stochastic formulation [23]. However, only $\sim 70$ of these e-foldings could fit in our local Hubble patch today, and any effect on the power spectrum due to the back reaction of the super-Hubble modes is inaccessible to observations.

Another possibility is that the inflaton potential may not be smooth. The observational bounds on $\Delta_{\mathcal{R}}^{2}$ hold from the observable horizon $\left(\sim 10^{4} \mathrm{Mpc}\right)$ down to the scale of the Lyman- $\alpha$ forest ( $\sim 1 \mathrm{Mpc}$ ) [24], constraining only about 10 e-foldings in the inflaton potential. An unusually flat portion in the inflaton potential (see Fig. 1) may yield a significantly larger amplitude of fluctuations, e.g. $\Delta_{\mathcal{R}}^{2} \sim 0.1$, and be inaccessible to cosmological observations if it happens at small enough scales. However, such a large amplitude may result in the production of Primordial Black Holes (PBHs), as the modes enter the Hubble radius, which results in further constraints on $\Delta_{\mathcal{R}}$.

To study the production of $\mathrm{PBHs}$ 25, let us look at the comoving scale of $R \lesssim 100 \mathrm{kpc}$, with the primordial amplitude of fluctuations $\Delta_{\mathcal{R}}$. This scale enters the Hubble radius when $a H \sim \pi / R$, which happens at redshift $z$, roughly given by

$$
z \sim \frac{\pi \sqrt{z_{e q}}}{H_{0} R \sqrt{\Omega_{m}}} \sim 10^{9} R^{-1}(\mathrm{kpc}),
$$

where $H_{0} \simeq 70 \mathrm{~km} / \mathrm{s} / \mathrm{Mpc}$ and $\Omega_{m} \simeq 0.3$ are today's Hubble constant and density parameter respectively, while $z_{e q} \sim 3 \times 10^{3}$ is the redshift of radiationmatter equality. As the modes of scale $R$ enter the Hubble radius, a fraction, $f$, of the Hubble patches have a fluctuation amplitude larger than 1, and thus may form PBHs, where $f$ is given by

$f\left(\Delta_{\mathcal{R}}\right) \sim \int_{1}^{\infty} \exp \left[-\frac{\zeta^{2}}{2 \Delta_{\mathcal{R}}^{2}}\right] \frac{d \zeta}{\sqrt{2 \pi \Delta_{\mathcal{R}}^{2}}} \sim \Delta_{\mathcal{R}} \exp \left[-\Delta_{\mathcal{R}}^{-2} / 2\right]$.

The current density in PBHs in units of the critical density, $\Omega_{\mathrm{PBH}}$, is given by

$$
\Omega_{\mathrm{PBH}} \simeq f\left(\Delta_{\mathcal{R}}\right)\left(\frac{z}{z_{e q}}\right) \epsilon,
$$

where $10^{-4}<\epsilon<1$, is the fraction of the mass in a Hubble patch that goes into the $\mathrm{PBH}$ [26]. Requiring that $\Omega_{\mathrm{PBH}}<\Omega_{m}$ yields

$$
f\left(\Delta_{\mathcal{R}}\right)<\frac{z_{e q} \Omega_{m}}{z \epsilon} \lesssim 10^{-2} R(\mathrm{kpc}) .
$$

For $\Delta_{\mathcal{R}}^{2}=0.1$, Eq. (44) gives $f\left(\Delta_{\mathcal{R}}\right) \sim 10^{-3}$, and thus Eq. (46), in combination with large scale structure observations, require

$$
0.1 \mathrm{kpc} \lesssim R \lesssim 100 \mathrm{kpc}
$$

Other astrophysical observations may put tighter constraints on a population of PBHs in the universe [27]. Instead of going through these constraints, let us give an example. Assume at $R=10 \mathrm{kpc}, \Delta_{\mathcal{R}}^{2}=0.1$ and $\epsilon=10^{-4}$. Using Eq. (45), $\Omega_{\mathrm{PBH}} \sim 0.01 \Omega_{m}$, while the PBH mass, $M_{\mathrm{PBH}}$ is $\epsilon M_{H}$, with $M_{H}$, being the Hubble mass at the Hubble entry:

$$
M_{H} \sim 10^{17} M_{\odot}\left(\frac{z_{e q}}{z}\right)^{2} \sim\left(10^{5} M_{\odot}\right) R^{2}(\mathrm{kpc}) .
$$

Therefore, with our choice of parameters, about $1 \%$ of the mass of the universe will end up in $\sim 10^{3} M_{\odot}$ black holes. This is consistent with current astrophysical constraints on such objects 27]. 
To summarize this section, we showed that if we assume a smooth inflaton potential, in single matter field models infrared back reaction (during the last 70 efoldings of inflation) should be negligible, at least to the 2nd order (one loop) in the amplitude of perturbations. However, if we relax this assumption, it is possible to construct an inflaton potential that allows a significant back reaction effect during the slow-roll inflationary period, without breaking any observational constraints.

\section{CONCLUSIONS}

We use a stochastic coarse-graining method to approach the problem of infrared back reaction in a single scalar field inflationary cosmology. Using the coarse-grained generalized Bardeen parameter, we selfconsistently derive the stochastic Langevin equation that governs the evolution of the local expansion rate. Solving this equation to second order (one loop) for chaotic inflation with a power law potential, we see that there is a non-vanishing average back reaction term due to the infrared modes that have left the Hubble radius. Although, the size of this effect will be negligible (within the observable universe) for smooth inflaton potentials, we show that it is possible to have non-smooth potentials which allow significant back reaction happening on sub-horizon scales, without breaking any observational constraints. A possible implication of such non-smooth potentials is the generation of a population of massive primordial black holes, which could be further used to constrain or verify these models.

\section{Acknowledgments}

We would like to thank Robert Brandenberger for many useful insights during the course of this research. G.G. would like to thank Brown University for a Dissertation Fellowship which this research was in part supported with.
[1] V. Mukhanov, H. Feldman, and R. Brandenberger, Phys. Rep. 215, 203 (1992).

[2] R. H. Brandenberger, arXiv:hep-th/0306071

[3] D. Brill and J. Hartle, Phys. Rev. 135, B271 (1964); R. Isaacson, Phys. Rev. 166, 1272 (1968).

[4] V. F. Mukhanov, L. R. Abramo and R. H. Brandenberger, Phys. Rev. Lett. 78, 1624 (1997) arXiv:gr-qc/9609026.

[5] L. R. Abramo, R. H. Brandenberger and V. F. Mukhanov, Phys. Rev. D 56, $3248 \quad$ (1997) arXiv:gr-qc/9704037.

[6] L. R. Abramo and R. P. Woodard, Phys. Rev. D 60, 044010 (1999) arXiv:astro-ph/9811430.

[7] W. Unruh, arXiv:astro-ph/9802323

[8] L. R. Abramo and R. P. Woodard, Phys. Rev. D 65, 043507 (2002) arXiv:astro-ph/0109271.

[9] G. Geshnizjani and R. Brandenberger, Phys. Rev. D 66, 123507 (2002) arXiv:gr-qc/0204074.

[10] S. Rasanen, JCAP 0402, $003 \quad$ (2004) arXiv:astro-ph/0311257.

[11] Ghazal Geshnizjani and Robert Brandenberger, arXiv:hep-th/0310265

[12] L. R. Abramo and R. P. Woodard, Phys. Rev. D 65, 063515 (2002) arXiv:astro-ph/0109272.

[13] A. Starobinsky, Phys. Lett. 117B, 175 (1982)

[14] A. Starobinsky and Jun'ichi Yokoyama, Phys. Rev. D50, 6357 (1994)

[15] M. Sasaki, Prog. Theor. Phys. 76, 1036 (1986).

[16] V. Mukhanov, Sov. Phys. JETP, 67, 1297 (1988).

[17] J. Bardeen, Phys. Rev. D22, 1882 (1980).

[18] A. Liddle and D. Lyth, Phys. Rep. 231, 1 (1993).

[19] N. Afshordi and R. H. Brandenberger, Phys. Rev. D 63, 123505 (2001) arXiv:gr-qc/0011075.

[20] A similar conserved quantity is derived in D. S. Salopek, and J. R. Bond, Phys. Rev. D 42, 3936 (1990).

[21] A. Starobinski, in Current Topics in Field Theory, Quantum Gravity and Strings, eds. H.J. de Vega and N.
Sanchez, Lecture Notes in Physics, vol. 206, p. 107 (Springer, Heidelberg, 1986); D. S. Salopek, and J. R. Bond, Phys. Rev. D 43, 1005 (1991).

[22] H. V. Peiris, et al., Astrophys. J. Supp. 148, 213 (2003) arXiv:astro-ph/0302225.

[23] A. Linde, D. Linde, and A. Mezhlumian,Phys. Rev. D 49, 1783 (1994) arXiv:gr-qc/9306035.

[24] A.R.C. Croft, et al., Astrophys. J. 581, 20 (2002) arXiv:astro-ph/0012324.

[25] For a modern account of $\mathrm{PBH}$ formation calculations, see A.M. Green, A.R. Liddle, K.A. Malik, and M. Sasaki, arXiv:hep-th/0310265

[26] I. Hawke, and J.M. Stewart, Class. Quant. Grav. 19, 3687 (2002).

[27] J. Yoo, J. Chaname, and A. Gould, Astrophys. J. 601, 311 (2004) arXiv:astro-ph/0307437; N. Afshordi, P, McDonald, and D.N. Spergel, Astrophys. J. Lett. 574, 71 (2003) arXiv:astro-ph/0302035.

[28] This method has been recently used in [10] to investigate the back reaction of UV modes in late Universe.

[29] Back reaction of infrared modes for two scalar field models where one of the fields is the inflaton and the other one is the clock was investigated in 11].

[30] A similar method has been used in [14] in a different context to investigate the behavior of weakly self-interacting scalar field in a de Sitter back ground.

[31] The cosmological scale factor is denoted by $a(t)$, the Hubble expansion rate by $H$, and the scalar matter field by $\varphi$.

[32] For a more detailed discussion about calculation of this parameter see [9, 11].

[33] In linear perturbation theory, $\delta \phi$ is forced to be equal to $\delta \psi$ by the off-diagonal $G_{i j}$ equations for all theories in which the off-diagonal components of $T_{i j}$ vanish to the linear order. This is in particular the case for scalar field matter.

[34] This is the simplest example of a cutoff function. In gen- 
eral, one can use any function which is close to one for $k<a H$ and tends to zero as $k$ goes to infinity fast enough to eliminate the ultraviolet divergence. 